\title{
A Novel Method for Preparing Surface-Modified Fluocinolone Acetonide Loaded PLGA Nanoparticles for Ocular Use: In Vitro and In Vivo Evaluations
}

\author{
Alaa H. Salama, ${ }^{1}$ Azza A. Mahmoud, ${ }^{1,2}$ and Rabab Kamel ${ }^{1,3}$
}

Received 30 August 2015; accepted 6 November 2015; published online 20 November 2015

\begin{abstract}
Our objective was to prepare nanoparticulate system using a simple yet attractive innovated method as an ophthalmic delivery system for fluocinolone acetonide to improve its ocular bioavailability. Poly(lactic-co-glycolic acid) (PLGA) nanoparticles were prepared by adopting thin film hydration method using PLGA/poloxamer 407 in weight ratios of 1:5 and 1:10. PLGA was used in 75/25 and 50/50 copolymer molar ratio of DL-lactide/glycolide. Results revealed that using PLGA with lower glycolic acid monomer ratio exhibited high particle size (PS), zeta potential (ZP) and drug encapsulation efficiency (EE) values with slow drug release pattern. Also, doubling the drug concentration during nanoparticles preparation ameliorated its EE to reach almost $100 \%$. Furthermore, studies for separating the un-entrapped drug in nanoparticles using centrifugation method at $20,000 \mathrm{rpm}$ for 30 min showed that the separated clear supernatant contained nanoparticles encapsulating an important drug amount. Therefore, separation of un-entrapped drug was carried out by filtrating the preparation using $20-25 \mu \mathrm{m}$ pore size filter paper to avoid drug loss. Aiming to increase the PLGA nanoparticles mucoadhesion ability, surface modification of selected formulation was done using different amount of stearylamine and chitosan $\mathrm{HCl}$. Nanoparticles coated with $0.1 \%$ w/v chitosan $\mathrm{HCl}$ attained most suitable results of PS, ZP and EE values as well as high drug release properties. Transmission electron microphotographs illustrated the deposition of chitosan molecules on the nanoparticles surfaces. Pharmacokinetic studies on Albino rabbit's eyes using HPLC indicated that the prepared novel chitosan-coated PLGA nanoparticles subjected to separation by filtration showed rapid and extended drug delivery to the eye.
\end{abstract}

KEY WORDS: chitosan $\mathrm{HCl}$; filtration; fluocinolone acetonide; nanoparticles; ocular; poloxamer; poly(lactic-co-glycolic acid); stearylamine.

\section{INTRODUCTION}

The sophisticated system of the eye presents a high resistance to all external substances including medications $(1,2)$. Great efforts have been devoted to increase ocular drug retention and ameliorate local as well as topical therapeutic effects and to overcome drawbacks of conventional delivery systems. This is crucial upon long-term use which is common in case of corticosteroids.

The anti-inflammatory corticosteroid drug, fluocinolone acetonide (FA), is used for the treatment of intermediate uveitis, posterior uveitis and panuveitis uveitis (3). Fluocinolone acetonide has low water solubility $(0.06 \mathrm{mg} / \mathrm{ml})$ (4) which is about only $4 \%$ of that for dexamethasone (5). The partition coefficient ( $n$-octanol/water; $\log \mathrm{P}$ ) of this drug is 3.19 (6).

Iluvien ${ }^{\circledR}$ (Alimera Sciences Inc., Alpharetta, GA, USA) is an intravitreal, microimplant containing fluocinolone acetonide. This

\footnotetext{
${ }^{1}$ Department of Pharmaceutical Technology, National Research Center, Dokki, Al-Bohooth St., Cairo, Egypt.

${ }^{2}$ Department of Pharmaceutical Technology, Faculty of Pharmaceutical Sciences and Pharmaceutical Industries, Future University in Cairo, Cairo, Egypt.

${ }^{3}$ To whom correspondence should be addressed. (e-mail: drrababk@hotmail.com)
}

implant is composed from the non-bioerodable polymer polyim-

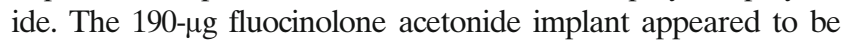
efficacious in the treatment of the patients with chronic diabetic macular edema (7). This implant provides substantial visual benefit by delivering the drug to the posterior eye tissue for up to 3 years (8). But unfortunately, patients receiving the fluocinolone acetonide implant are at higher risk of developing an increase in their intraocular pressure which is a major potential complication for this dosage form (9), in addition to the patient incompliance which may be encountered with the implant administration.

Oral corticosteroids are associated with numerous systemic side effects that may be limit their use and in some cases may be severe and life-threatening drugs $(10,11)$. Therefore, non-invasive ocular delivery systems for fluocinolone acetonide with minimal side effects are recommended to deliver the drug in a controlled manner.

For ophthalmic applications, properly formulated drugloaded colloidal particles are reported to provide prolonged retention and less frequent need of application in addition to the well-known advantages of eye drops (12).

In a previous literature, FA was complexed with several types of cyclodextrins $(\alpha-, \beta-$ and hydroxypropyl- $\beta-C D)$ and then it was incorporated into multivesicular liposomes. The opti-

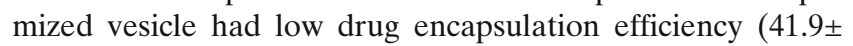


$1.8 \%$ ) and showed drug release over only $3 \mathrm{~h}$. This formulation was intended to be used for the treatment of ocular inflammatory disease but no pharmacological study was adopted (13).

Biodegredable polymers, like poly(lactic-co-glycolic acid) (PLGA), are widely used because of their desirable properties including safety, biodegradability, suitability for different hydrophilic and hydrophobic types of drugs, ability to provide sustained drug release as well as drug targeting to specific organs (14). PLGA nanoparticles can generally be prepared using solvent evaporation procedure. The resultant nanoparticles provide slow drug release depending on the hydrolysis rate of the polymer (15). PLGA chemical structure is composed of different ratios of lactic acid and glycolic acid which provides a large range of physicochemical properties depending on lactic/glycolic acid ratio. During design and formulation, the selection of the suitable type of PLGA is crucial for successful drug delivery (16).

Although PLGA nanoparticles attained good results in many studies, the lack of mucoadhesiveness caused unsuccessful results (17). Therefore, in this study, we tried to apply some formulation modifications to overwrite any PLGA nanoparticles' inherent disadvantages.

Pluronic ${ }^{\circledR}$ block copolymers are amphiphilic synthetic polymers built of hydrophilic part, poly(ethylene oxide) (PEO) blocks, and hydrophobic part, poly(propylene oxide) (PPO) blocks, arranged in triblock structure: PEO-PPOPEO. Poloxamer 407 (Pluronic $\AA$ acid F127) is composed of $70 \%$ polyoxyethylene units and $30 \%$ polyoxypropylene blocks (18). The preparation of mixed PLGA-poloxamers nanoparticles was investigated by some literature aiming to ameliorate particle stability and drug release pattern (19).

In the conventional method for PLGA nanoparticles preparation, particles are collected after evaporation of the organic solvent used to dissolve the ingredients. However, this method has several disadvantages comprising the need to use and to evaporate high amount of organic solvents as well as low drug encapsulation efficiency in many cases (20). Therefore, in our study, we explored a novel method trying to avoid these disadvantages.

In this study, fluocinolone acetonide loaded PLGA nanoparticles were prepared using basically a three-component mixture of polymer/surfactant/drug (PLGA, poloxamer and FA). The fluocinolone acetonide loaded PLGA nanoparticles were prepared using the thin film hydration method. The innovated polymeric nanoparticulate (NP) was subjected to characterization, surface-modification as well as in vitro and in vivo evaluations.

\section{MATERIALS AND METHODS}

\section{Materials}

Fluocinolone acetonide was obtained from Inspire Pharma, Egypt. Chitosan $\mathrm{HCl}$ (Cs) was kindly gifted from Zhejiang Chemicals Import \& Export Cooperation, China. Stearylamine (SA) was purchased from Sigma-Aldrich, Barcelona, Spain. Poly(lactic-co-glycolic acid) with an acid terminated grade copolymer and with an inherent viscosity midpoint of $0.2 \mathrm{dl} / \mathrm{g}$ : Purasorb® PDLG 5002A, 50/50 molar ratio of DL-lactide/ glycolide copolymer (P 5002) and Purasorb® PDLG 7502A, $75 / 25$ molar ratio of DL-lactide/glycolide copolymer (P 7502), was obtained as free samples from Purac Biomaterials, Gorinchem, Netherlands. Poloxamer 407 (Pluronic ${ }^{\circledR}$ acid
F127) was obtained from Sigma-Aldrich Chemie GmbH, Germany. Cellulose membrane (molecular weight cut-off 12,000 g/ mole) was purchased from Sigma Chemical Company, USA. Porcine mucin was obtained from Sigma-Aldrich Chemie $\mathrm{GmbH}$, Japan. Potassium dihydrogen phosphate and disodium hydrogen phosphate were purchased from Sisco Research Laboratories Pvt. Ltd., India. HPLC grade of acetonitrile was purchased from SDFCL-SD Fine-Chem Limited, India.

\section{Methods}

\section{Preparation of PLGA Nanoparticles}

Fluocinolone acetonide loaded PLGA nanoparticles were prepared by an innovated simple thin film hydration method in concentrations as mentioned in Table I. Briefly, the drug, PLGA and poloxamer were dissolved in $20 \mathrm{ml}$ of acetonitrile in a clean and dry round bottom flask. Then the rotator evaporator (Heidolph Instruments $\mathrm{GmbH}$, Germany) was used to remove the organic solvent. The thin film formed after solvent evaporation was then hydrated by $10 \mathrm{ml}$ phosphate buffer $(\mathrm{pH}$ 7.4). The non-encapsulated drug crystals were eliminated by filtration through Whatman ${ }^{\circledR}$ filter paper No 41 (pore size of 20-25 $\mu \mathrm{m}$; England) (21). Following the same procedure, poloxamer system (FP) was prepared using only poloxamer and fluocinolone acetonide (without the use of PLGA).

\section{Characterization of the Fluocinolone Acetonide Loaded PLGA Nanoparticles}

PLGA Nanoparticles Size and Zeta Potential. Zetasizer Nano ZS (Malvern Instruments, UK) was used to evaluate the PLGA nanoparticles size and zeta potential. The average particle size and size distribution (polydispersity index; PDI) were measured by dynamic light scattering at 173 and $25^{\circ} \mathrm{C}$. The zeta potential was measured by laser Doppler anemometry at $25^{\circ} \mathrm{C}$ using M3-PALS technology.

Table I. Composition of Fluocinolone Acetonide Loaded PLGA Nanoparticles

\begin{tabular}{|c|c|c|c|}
\hline \multirow{2}{*}{$\begin{array}{l}\text { Formula } \\
\text { code }\end{array}$} & \multicolumn{3}{|l|}{ Composition } \\
\hline & $\begin{array}{l}50 \mathrm{mg} \text { of } \\
\text { PLGA type }\end{array}$ & $\begin{array}{l}\text { Poloxamer } 407 \\
\text { concentration } \\
(\mathrm{mg})\end{array}$ & $\begin{array}{l}\text { Drug } \\
\text { concentration } \\
(\mathrm{mg})\end{array}$ \\
\hline $\mathrm{F} 1$ & P $5002^{a}$ & 250 & 5 \\
\hline $\mathrm{F} 2$ & P $5002^{a}$ & 500 & 5 \\
\hline F3 & P $7502^{b}$ & 250 & 5 \\
\hline F4 & P $7502^{b}$ & 500 & 5 \\
\hline F5 & P $5002^{a}$ & 250 & 10 \\
\hline F6 & $\mathrm{P} 5002^{a}$ & 500 & 10 \\
\hline F7 & P $7502^{b}$ & 250 & 10 \\
\hline F8 & P $7502^{b}$ & 500 & 10 \\
\hline
\end{tabular}

${ }^{a}$ P 5002: 50/50 DL-lactide/glycolide copolymer ${ }^{b}$ P 7502: 75/25 DL-lactide/glycolide copolymer 
Determination of Encapsulation Efficiency of PLGA Nanoparticles. Drug-loaded nanoparticles were subjected to centrifugation (Sigma 3-30K, Spincontrol Comfort, Germany) at $20,000 \mathrm{rpm}$ for $30 \mathrm{~min}$. Acetonitrile was added to the collected nanoparticles (residue) and sonication for $10 \mathrm{~min}$ was done. The obtained clear supernatant was assessed for drug content spectrophotometrically at $234.7 \mathrm{~nm}$ using Shimadzu UV spectrophotometer (2401/PC, Japan).

The encapsulation efficiency values were calculated using the following equation:

Encapsulation efficiency $(\%)=($ Amount of encapsulated drug/Total amount of added drug $) \times 100$

Determination of Loading Capacity of PLGA Nanoparticles. Accurately volumes of PLGA nanoparticles were dried using freeze drying (Christ freeze dryer, ALPHA 2-4 LD plus, Germany). After that, the dried PLGA nanoparticles were weighed. The loading capacity (LC) for the PLGA nanoparticles was calculated according to the following equation:

Loading capacity $(\%)=($ Amount of encapsulated drug/PLGA nanoparticles weight $) \times 100$

In Vitro Drug Release Studies from PLGA Nanoparticles. Colloidal preparations (equivalent to $1 \mathrm{mg}$ of drug) were put in a dialysis bag which was tightly closed from both ends before dipping in the release medium $(20 \mathrm{ml}$ phosphate buffer $\mathrm{pH} 7.4$ containing $10 \%$ ethanol to obtain sink conditions). The release media was kept at $34 \pm 0.5^{\circ} \mathrm{C}$ and was operated at 100 storks per minute. This assembly was secured against any loss of release medium. Withdrawn samples $(2 \mathrm{ml})$ at specified time intervals during $24 \mathrm{~h}$ were spectrophotometrically analysed for fluocinolone acetonide content at $240.4 \mathrm{~nm}$ (Shimadzu UV spectrophotometer, 2401/PC, Japan). Fresh release medium was added to replenish withdrawn samples.

Decisive release parameters include the following: cumulative percentage of drug released at $1 \mathrm{~h}\left(Q_{1 \mathrm{hr}}\right)$, cumulative percentage of drug released at $24 \mathrm{~h}\left(Q_{24 \mathrm{hr}}\right)$, release efficiency (RE) and mean release time (MRT).

The RE is calculated from the area under the dissolution curve (AUC) at time $t$ using the trapezoidal rule according to the following equation (22):

$\mathrm{RE}=\left(\int_{0}^{t} y \times \mathrm{d} t / y_{100} \times t\right) \times 100$

where $y$ is the amount of drug dissolved at time $t$.

To characterize the drug-release process, the MRT was calculated according to the following equation (23):

$$
\operatorname{MRT}=\frac{\sum_{i=1}^{i=n} t_{\mathrm{mid}} \times \Delta M}{\sum_{i=1}^{i=n} \Delta M}
$$

where $i$ is the release sample number, $n$ is the number of release sample time, $t_{\text {mid }}$ is the time at the midpoint between $i$ and $i^{-1}$ and $\Delta M$ is the additional amount of drug dissolved between $i$ and $i-1$. A higher value of MRT means a lower release rate (24).

Preparation and Evaluation of Surface-Modified PLGA Nanoparticles.

Stearylamine (SA)-modified PLGA nanoparticles

Using the same procedure listed previously for the preparation of the nanoparticle systems using the thin film hydration technique, stearylamine modified nanoparticles where prepared by dissolving different amounts of stearylamine (SA: $5,10,25$ or $50 \mathrm{mg}$ ) in the acetonitrile during the preparation of the selected fluocinolone loaded nanoparticles.

\section{Chitosan HCl surface modified PLGA nanoparticles}

The selected PLGA nanoparticles were prepared using the thin film hydration technique as stated previously. After that chitosan $\mathrm{HCl}$ (Cs: $0.1,0.25$ or $0.5 \% \mathrm{w} / \mathrm{v}$ ) was added to the final PLGA nanoparticles suspension and then mixed at $300 \mathrm{rpm}$ for 30 min using magnetic stirrer (Thermolyne Corp., USA) at room temperature.

\section{Characterization and Evaluation of Surface Modified PLGA Nanoparticles.}

Differential scanning calorimetry (DSC)

DSC was used to inspect the thermal properties of the pure drug, PLGA, poloxamer, physical mixtures and selected PLGA nanoparticles using Shimadzu differential scanning calorimeter (DSC-60, Shimadzu, Japan). Samples (2 mg) were sealed in a standard aluminum pan and scanned at a constant heating rate of $5^{\circ} \mathrm{C} / \mathrm{min}$ at temperature range from 0 to $350^{\circ} \mathrm{C}$ under constant purging of argon $(20 \mathrm{ml} / \mathrm{min})$.

\section{PLGA nanoparticles shape}

Transmission electron microscope (TEM) was used to examine the shape of the selected PLGA nanoparticles and surface modified PLGA nanoparticles (Jeol, JEM-1230, Japan). For sample preparation, nanoparticles were applied on a carbon 
coated copper grid and then stained with $2 \%$ phosphotungstic acid.

In vitro drug release studies for fluocinolone acetonide loaded PLGA nanoparticles

The release of fluocinolone acetonide from the selected PLGA nanoparticles and surface-modified PLGA nanoparticles was compared according to the method described previously.

Assessment of the mucoadhesive properties of surface modified PLGA nanoparticles

The interaction between the oppositely charged mucin and chitosan $\mathrm{HCl}$ molecules coated PLGA nanoparticles is the basis of this test. Thus, a solution of mucin $(0.4 \mathrm{mg} / \mathrm{ml})$ in phosphate buffer ( $\mathrm{pH}$ 7.4) was prepared and mixed with selected PLGA nanoparticles and surface-modified nanoparticles $(1: 1 \mathrm{v} / \mathrm{v})$, at $37^{\circ} \mathrm{C}$. Zeta potential and particle size of both solutions were measured before and after thorough mixing $(25,26)$.

\section{In Vivo Evaluation of Selected Fluocinolone Acetonide Loaded PLGA Nanoparticles}

Male healthy Albino rabbits $(1.5-2.0 \mathrm{~kg})$ were kept under standardized conditions and were randomly segregated into different groups. Animals' management and studies method were based on local, national, ethical and regulatory principles for animal care. The followed protocol was approved by the Research Ethical Committee of the Faculty of Pharmaceutical Sciences and Pharmaceutical Industries at Future University in Egypt (approval number REC-FPSPI-1/1).

\section{Ocular Irritation Study}

Two groups of Albino rabbits $(n=6)$ received on their right eye $50 \mu \mathrm{l}$ of the selected PLGA nanoparticles (group 1) or the surface-modified PLGA nanoparticles (group 2), while the left eye was used as a control. Application of the tested formulation onto the rabbit's cornea was repeated every $2.5 \mathrm{~h}$ through a period of $7.5 \mathrm{~h}$ per day for three successive days and once on the fourth day (27).

After 1 and $24 \mathrm{~h}$ from last instillation, eyes were examined under general anesthesia $(35 \mathrm{mg} / \mathrm{kg}$ ketamine and $5 \mathrm{mg} / \mathrm{kg}$ xylazine) and were evaluated according to Draize technique (28). The eyelids, cornea, iris, conjunctiva and anterior chamber were examined for inflammation or toxic reaction. The evaluation criteria used was as follows: $0-3.9$ was considered to be nonirritant, 4-8.9 was considered to be slightly irritant, 9-12.9 moderately irritant and 13-16 seriously irritant (29).

\section{Pharmacokinetic Study}

Study Design. A single dose randomized crossover design was performed. Six Albino rabbits were treated with single unilateral eye drops $(50 \mu \mathrm{l})$ of the selected fluocinolone acetonide loaded PLGA nanoparticles (group 1) or surfacemodified fluocinolone acetonide loaded PLGA nanoparticles (group 2). After tested formulation application, tear samples were collected at specified time intervals using five sterile 6- mm-diameter filter paper discs (Whatman ${ }^{\circledR}$ no. $1,11 \mu \mathrm{m}$ pore size, England). The filter paper discs were placed under the eyelid of rabbits for $1 \mathrm{~min}$. Then the discs were vortex for $5 \mathrm{~min}$ in $100 \mu \mathrm{l}$ acetonitrile spiked with $1 \mu \mathrm{l}$ of the internal standard fluticasone propionate. After that, samples were stored in Eppendorf tubes ${ }^{\circledR}$ (UK) for further processing (30).

Chromatographic Conditions. Before the determination of fluocinolone acetonide concentrations in tears, an accurate, sensitive and selective method for HPLC was developed and validated.

HPLC system consisting of isocratic pump LC-10 AD and a UV/vis detector SPD-10A connected to a SCL-10A integrator, Knauer Advanced Scientific UV 2250 (Berlin, Germany). The analytical column was Eurosphere C18 column, $250 \mathrm{~mm} \times 4.6$ I.D. $\mathrm{mm}$, particle size $5 \mu \mathrm{m}$ (Berlin, Germany). The isocratic mobile phase comprised acetonitrile and $20 \mathrm{mM}$ potassium dihydrogen phosphate buffer $(65: 35, v / v)$ of $\mathrm{pH} 2.6$. The mobile phase was filtered and degassed. The flow rate was set at $1.2 \mathrm{ml} / \mathrm{min}$ and the column effluent was monitored continuously using UV detection at $254 \mathrm{~nm}$. The tested samples were injected in the HPLC system in a volume of $20 \mu \mathrm{l}$.

\section{Statistical Analysis}

SPSS ${ }^{\circledR}$ software (USA) was used for statistical analysis of the data. Unpaired $t$ test or one-way analysis of variance test (ANOVA) followed by the least significant difference test (LSD) were used to compare differences between two or more groups, respectively.

During the in vitro evaluation of the preparations, data were collected from two different batches. The values of means and standard deviations were obtained from duplicate test results for each batch.

\section{RESULTS AND DISCUSSION}

The utility of adding poloxamers to the PLGA nanoparticles is far beyond the stabilization of the emulsion during preparation. The obtained nanoparticles composed of physically entangled PLGA molecules with PEO moieties in the poloxamer can allow better drug protection and more tailored drug release. Based on these advantages, in the present study we aimed to investigate the potential of some selected blends of PLGA and poloxamer 407 (Pluronic ${ }^{\circledR}$ F127) to formulate suitable PLGA nanoparticles, by varying PLGA type, PLGA/ poloxamer weight ratio as well as drug concentration. Composition of the designed formulations is listed in Table I.

After nanoparticles formulations, they are subjected to centrifugation followed by washing to remove process impurities as well as un-entrapped drug using an appropriate solvent (31). However, caking is a great disadvantage of this process because of the difficulty to redisperse particles in the sediment. In addition, a certain portion of the product in the supernatant is probably lost. Also, sometimes it is difficult to select suitable solvent which can dissolve un-entrapped drug crystals without affecting the prepared nanoparticles. Therefore, to avoid all these complications, a simple and reproducible method was used in our study to separate un-entrapped 
drug by filtering the preparations using Whatman ${ }^{\circledR}$ filter paper No 41 (pore size of 20-25 $\mu \mathrm{m}$; England) (21).

After the preparation of fluocinolone acetonide loaded PLGA nanoparticles, un-entrapped drug crystals were removed by filtration and were then subjected to further characterizations. In a second step, the filtered PLGA nanoparticles were then subjected to the habitual nanoparticles separation process by subjecting them to centrifugation at 20,000 rpm for $30 \mathrm{~min}$ to guarantee successful separation. After that, full investigations of the residue as well as the supernatant were done. This can allow the examination of the benefit of the separation of un-entrapped drug by the filtration process only and, hence, the investigation of the advantages versus disadvantages of separation of prepared nanoparticles systems using centrifugation.

Furthermore, in order to determine the feasibility of preparing PLGA nanoparticles using poloxamer 407, fluocinolone acetonide loaded poloxamer system (FP) was prepared as discussed in the experimental part, for comparing its properties with the innovated PLGA nanoparticles ocular delivery system.

\section{Characterization of Fluocinolone Acetonide Loaded PLGA Nanoparticles}

The filtrated PLGA nanoparticles were subjected to TEM and particle size analysis. Furthermore, after centrifugation of the PLGA nanoparticles, the obtained clear supernatant-which is an indication of successful nanoparticles separation-was subjected to TEM and particle size analysis to examine the presence of drug loaded PLGA nanoparticles in it.

TEM investigation for the filtrated PLGA nanoparticles revealed the absence of drug crystals of any un-entrapped drug, proving the potential use of the filtration method under investigation for separation of drug loaded nanoparticles from un-entrapped drug crystals (Fig. 1). Furthermore, TEM and particle size analysis for the clear supernatant obtained after the habitual separation by centrifugation prove the presence of nanoparticles in the size range from 85 to $160 \mathrm{~nm}$ (Fig. 1 and Table II). This can demonstrate the risk of losing certain amount of drug loaded nanoparticles due to separation by high speed centrifugation.

TEM photographs showed that PLGA nanoparticles had an almost circular or quadratic outline; it can be assumed that the presence of poloxamer in the PLGA nanoparticles can alter the geometry of the dispersed nanoparticles (Fig. 1). It was reported by Salama et al. that poloxamer 407 was able to alter the spherical shape of phospholipid-based colloidal nano-vesicles to cubic shape (32).

Regarding the effect of used additives on the particle size and zeta potential values of the obtained PLGA nanoparticles, it can be noticed from data listed in Table II that PLGA type has a great impact on recorded particle size values as well as zeta potential values for the prepared formulations. It was found that PLGA nanoparticles prepared using PLGA 7502 $(\mathrm{F} 3, \mathrm{~F} 4$ and F8) had greater particle size values $(p<0.05)$ compared to those for their corresponding PLGA nanoparticles prepared using PLGA P 5002 (F1, F2 and F6, respectively). This can be explained by that the glycolic acid monomer ratio in PLGA P 5002A and PLGA P 7502A (lactic/glycolic acid ratios: $50 / 50$ and $75 / 25$, respectively) affects the minimum content of PLGA required for the vesicular system to be
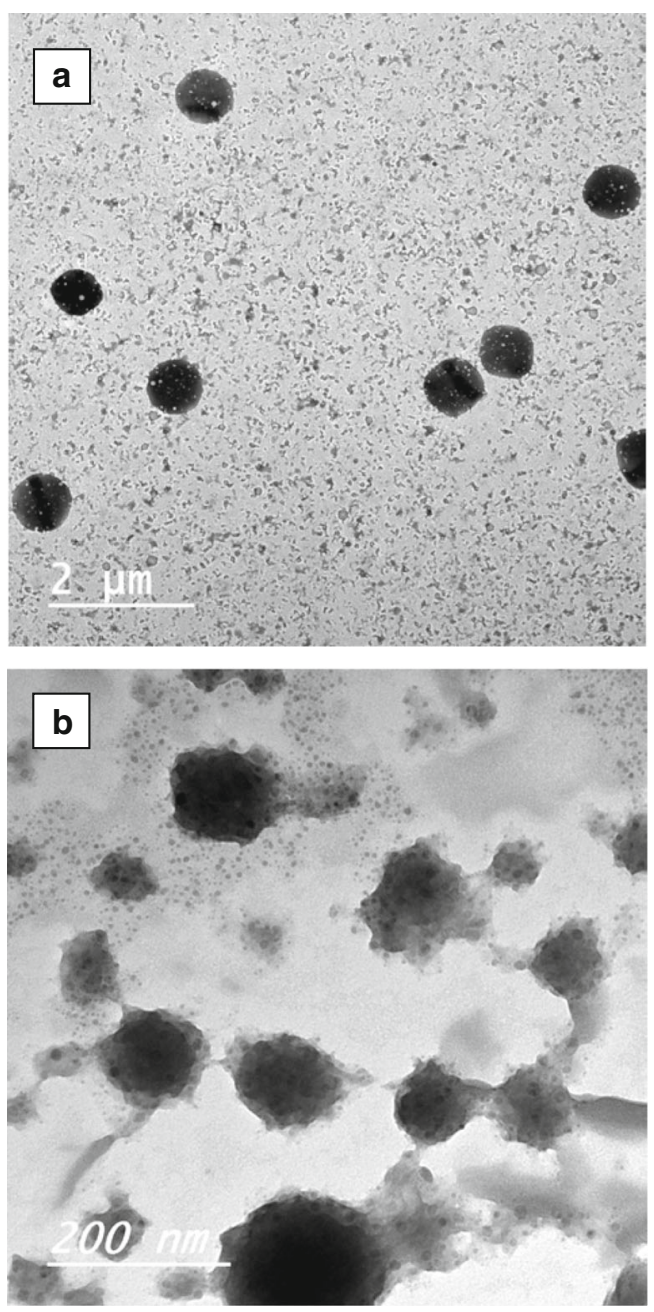

Fig. 1. TEM photographs of selected fluocinolone acetonide loaded PLGA nanoparticles (a) and its supernatant (b)

stable (16). It is generally known that the hydrophobic properties of PLGA are predominant; however, this hydrophobicity decreases by increasing the glycolic acid monomer in the copolymer, this can give the chance for easier interfacial arrangement of PLGA molecules (16).

This can explain the noticed smaller particles size and particle distribution values of nanoparticles prepared from PLGA P 5002 (F1, F2 and F6) as low amount of PLGA was needed to formulate the nanoparticles. Therefore, nanoparticles containing less amount of the bulk polymer, PLGA, are small in size due to tight packing of the nanoparticle components; this can be clearly seen in Table II. Furthermore, PLGA with high portion of glycolic acid monomer has more ability to reduce the system interfacial tension, resulting in particles with smaller size (16).

Table II reveals that by increasing the drug concentration from 5 to $10 \mathrm{mg}$ (F1-F4 vs F5-F8, respectively) had no significant effect on particle size or zeta potential values $(p>0.05)$. It was denoted that increasing poloxamer amount from 250 to $500 \mathrm{mg}$ led to increase in nanoparticle size $(p<0.05)$; this can be noticed while comparing each formula versus its corresponding one with lower poloxamer content (F1 vs F2, F3 vs F4 and F7 vs F8). This can be expected due the accumulation of a larger amount of poloxamer molecules within the formed nanoparticles and/or 
Table II. Particle Size, Polydispersity Index and Zeta Potential Values for Fluocinolone Acetonide Loaded PLGA Nanoparticles

\begin{tabular}{|c|c|c|c|c|c|c|}
\hline \multirow[t]{2}{*}{ Formula code } & \multicolumn{2}{|l|}{ Particle size (nm) } & \multicolumn{2}{|l|}{ PDI } & \multicolumn{2}{|c|}{ Zeta potential $(\mathrm{mV})$} \\
\hline & Total preparation & Preparation supernatant & Total preparation & $\begin{array}{l}\text { Preparation } \\
\text { supernatant }\end{array}$ & $\begin{array}{l}\text { Total } \\
\text { preparation }\end{array}$ & $\begin{array}{l}\text { Preparation } \\
\text { supernatant }\end{array}$ \\
\hline $\mathrm{F} 1$ & $159.43 \pm 1.55$ & $111.46 \pm 0.45$ & $0.24 \pm 0.00$ & $0.20 \pm 0.01$ & $-5.24 \pm 0.54$ & $5.25 \pm 0.64$ \\
\hline $\mathrm{F} 2$ & $203.17 \pm 4.55$ & $178.6 \pm 2.26$ & $0.38 \pm 0.02$ & $0.19 \pm 0.01$ & $-2.53 \pm 0.23$ & $4.87 \pm 0.58$ \\
\hline F3 & $217.97 \pm 7.77$ & $85.17 \pm 0.66$ & $0.51 \pm 0.10$ & $0.16 \pm 0.01$ & $-5.83 \pm 1.36$ & $4.66 \pm 0.18$ \\
\hline $\mathrm{F} 4$ & $453.83 \pm 20.06$ & $109.27 \pm 1.50$ & $0.59 \pm 0.03$ & $0.27 \pm 0.01$ & $-8.40 \pm 0.73$ & $4.03 \pm 0.38$ \\
\hline F5 & $186.17 \pm 10.76$ & $100.19 \pm 2.02$ & $0.42 \pm 0.05$ & $0.21 \pm 0.01$ & $-5.02 \pm 0.32$ & $5.02 \pm 0.51$ \\
\hline F6 & $148.00 \pm 1.87$ & $159.93 \pm 1.43$ & $0.25 \pm 0.00$ & $0.17 \pm 0.02$ & $-5.80 \pm 0.90$ & $4.35 \pm 0.13$ \\
\hline F7 & $131.00 \pm 0.44$ & $89.61 \pm 0.90$ & $0.23 \pm 0.01$ & $0.17 \pm 0.01$ & $-6.21 \pm 1.19$ & $3.73 \pm 0.81$ \\
\hline F8 & $274.23 \pm 30.36$ & $106.63 \pm 2.19$ & $0.38 \pm 0.03$ & $0.20 \pm 0.01$ & $-2.21 \pm 0.10$ & $2.18 \pm 0.11$ \\
\hline FP & $2298.67 \pm 108.52$ & $34.33 \pm 0.33$ & $0.91 \pm 0.06$ & $0.52 \pm 0.03$ & $-2.88 \pm 0.26$ & $-1.43 \pm 0.24$ \\
\hline
\end{tabular}

$P D I$ polydispersity index

on their surfaces. This phenomenon can be attributed to the increase of polymer chains number per unit volume which led to a more bulky structure and thus colloidal system with larger particle size.

The relatively low polydispersity index values (Table II) are evidencing the formation of an almost homogeneous population of nanoparticles in the whole preparation and the supernatant as well. This could be attributed to that poloxamer 407 was arranged on the surface of the nanoparticles with its hydrophilic moieties (PEO) facing the aqueous solution. Such arrangement of the poloxamer moieties provides a shielding barrier which prevents the aggregation of nanoparticles and stabilizes them (33-35). Therefore, the resulted nanoparticles were relatively small in size with a somewhat uniform particle size distribution (small PDI values).

Zeta potential values for all the formulated PLGA nanoparticles were negative in charge. This was expected due to the presence of terminal carboxylic acid groups in PLGA molecules. The deposition of a certain fraction of poloxamer on nanoparticles surface can result in lower negative values than those reported for classical PLGA nanoparticles in previous literature (36).

Increasing the amount of poloxamer from 250 to $500 \mathrm{mg}$ resulted in decreasing the negative zeta potential value of the nanoparticles $(p<0.05)$; this can be clearly seen while considering F1 versus $\mathrm{F} 2$ as well as F7 versus F8. On the other side, the positively charged zeta potential of small nanoparticles found in the supernatant can prove that these are mainly formed from poloxamer molecules.

The size and PDI values of the polymeric nanoparticles prepared without PLGA (FP) were significantly larger than those for PLGA nanoparticles (F1-F8; $p<0.05)$. This can be attributed to a certain interactions occurring between poloxamer and PLGA consisting mainly of hydrogen bonding between the $\mathrm{OH}$ groups of PLGA and the ether groups of poloxamer, which allow for more assembling and entanglement between monomers leading to more compact and smaller polymeric nanoparticles.

On the other side, the particle size recorded for the supernatant of fluocinolone acetonide was significantly smaller $(p<0.05)$ than other PLGA nanoparticles formulations indicating that the supernatants of the latter was composed from poloxamer in addition to PLGA as well.
Encapsulation Efficiency (EE) and Loading Capacity (LC) for Fluocinolone Acetonide Loaded PLGA Nanoparticles

Considering the data presented in Table III, we can detect that, generally, nanoparticles containing PLGA P 7502 $(\mathrm{F} 3, \mathrm{~F} 4$ and F8) attained higher EE values $(p<0.05)$ when compared with those for their corresponding ones prepared using PLGA P 5002 (F1, F2 and F6). This could be attributed to the higher lipophilicity of PLGA P 7502 which can be able to entrap the lipophilic drug, fluocinolone acetonide, and prevent its escape to the outer media. Janoria and Mitra detected similar results in case of formulating PLGA microspheres loaded with ganciclovir and its lipophilic prodrug (37). As a consequence, drug content was lower in nanoparticles present in the supernatant of the systems prepared using the latter type of PLGA as a larger drug amount was already entrapped in the residual nanoparticles.

Generally, formulations prepared with higher drug concentration attained higher encapsulation efficiency and loading capacity compared with the corresponding formulations prepared with lower drug concentration, this was noticed in case of both nanoparticles formulations and their supernatant $(p<0.05)$.

In conclusion, we can certitude that the clear supernatant obtained after centrifugation of the formulated PLGA nanoparticles contained a certain amount of drug encapsulated in small PLGA nanoparticles which have escaped in the

Table III. Encapsulation Efficiency and Drug Loading Values for Fluocinolone Acetonide Loaded PLGA Nanoparticles

\begin{tabular}{lllr}
\hline Formula code & \multicolumn{2}{l}{ Encapsulation efficiency (\%) } & Drug loading (\%) \\
\cline { 2 - 3 } & Residue & Supernatant & \\
\hline F1 & $41.85 \pm 4.07$ & $15.15 \pm 4.07$ & $6.92 \pm 0.63$ \\
F2 & $56.21 \pm 3.76$ & $6.48 \pm 3.77$ & $4.10 \pm 0.41$ \\
F3 & $78.09 \pm 0.76$ & $11.39 \pm 1.26$ & $16.53 \pm 1.30$ \\
F4 & $85.22 \pm 5.32$ & $18.57 \pm 5.26$ & $15.32 \pm 1.43$ \\
F5 & $68.28 \pm 0.76$ & $35.83 \pm 0.76$ & $8.04 \pm 0.82$ \\
F6 & $70.97 \pm 0.76$ & $30.92 \pm 0.76$ & $6.65 \pm 0.56$ \\
F7 & $91.94 \pm 1.52$ & $11.87 \pm 1.52$ & $15.55 \pm 1.19$ \\
F8 & $75.27 \pm 5.32$ & $26.77 \pm 5.32$ & $12.24 \pm 1.06$ \\
FP & $83.54 \pm 3.49$ & $20.64 \pm 3.49$ & \\
\hline
\end{tabular}


supernatant. Discard of such supernatant would result in loss of a significant amount of the drug that can reach $35 \%$ of the added drug in some cases (F5).

Therefore, purification of prepared nanoparticles from un-entrapped drug crystals can better be done by filtration through Whatman ${ }^{\circledR}$ filter paper No 41 (pore size of 20-25 $\mu \mathrm{m}$; England), this can assure the 'hold on' of almost all the amount of entrapped drug. The total high EE values (residue+supernatant) shown in Table III may indicate successful encapsulation of the lipophilic drug in the lipophilic PLGA nanoparticles and can focus on one of the benefits of usage of the suggested method of separation to remove only un-entrapped drug crystals and then to use the purified PLGA nanoparticles formulations without need of high speed centrifugation.

\section{In Vitro Drug Release Studies for Fluocinolone Loaded PLGA Nanoparticles}

The cumulative amount of drug released was plotted against time (Fig. 2) and release parameters were calculated to compare between investigated formulations. The investigated release parameters (Table IV) involved $Q_{1 \mathrm{hr}}, Q_{24 \mathrm{hr}}, \mathrm{RE}$ and MRT.

From the release data showed in Table IV, it can be clearly seen that formulations prepared using PLGA P 5002 (F1, F2, F5 and F6) attained higher drug release compared to their corresponding ones prepared using PLGA P 7502 (F3, F4, F7 and F8, respectively), this can be detected from both percentage drug released after $24 \mathrm{~h}$ as well as RE values $(p<0.05)$. This can be expected due to the high glycolic acid content in PLGA P 5002 (hydrophilic part) which increases its degradation rate when compared to PLGA polymers with lower glycolic content (PLGA P 7502) (38). Also, the generally large particle size values for nanoparticles prepared using PLGA P 7502 can result in a slower drug release pattern due to smaller surface area available for drug release.
The bimodal particle size range (Fig. 3) observed in all formulations (large-sized residual nanoparticles and smaller nanoparticles in the supernatant) allowed to obtain a more or less biphasic release pattern, having a relatively rapid release during first hours $\left(Q_{1 \mathrm{hr}}\right)$ followed by a decrease in drug release rate till $24 \mathrm{~h}$, this can be due to the fast escape of particles adsorbed on the nanoparticle external surfaces or entrapped in the outer layers. Also, small nanoparticles that were detected in the supernatant would provide fast release of their encapsulated drug.

However, there was no significant difference $(p>0.05)$ in the release between different formulations considering the MRT (Table IV).

On the other side, increasing drug or poloxamer concentration in PLGA nanoparticles did not have any significant effect on the calculated release data $(p>0.05)$.

Considering the previous interpretations, we can detect that among fluocinolone acetonide loaded nanoparticles consisted of PLGA/poloxamer blends, F2 and FP showed the highest $Q_{24 \mathrm{hr}}$ as well as RE values $(p<0.05)$. This fast drug release from FP can be explained on the basis that the hydrophilicity of poloxamer particles in the FP caused fast drug release. But, on the other hand, the high PDI values of the FP preparation are not favoured. The polydispersity index is a measure of the width of the particle size distribution; a high PDI value reflects a heterogeneous population, a PDI value larger than 0.5 indicates large size distribution as well as a high tendency for aggregation which negatively affects preparation stability (39). Therefore, F2, being the most suitable preparation, was selected for modification and further investigations.

\section{Evaluation of Surface-Modified Selected PLGA Nanoparticles}

Particle Size, Zeta Potential, Polydispersity Index and Encapsulation Efficiency. In a trial to impart positive charge to the selected PLGA nanoparticles (F2) and to increase its zeta potential value in order to increase its stability and

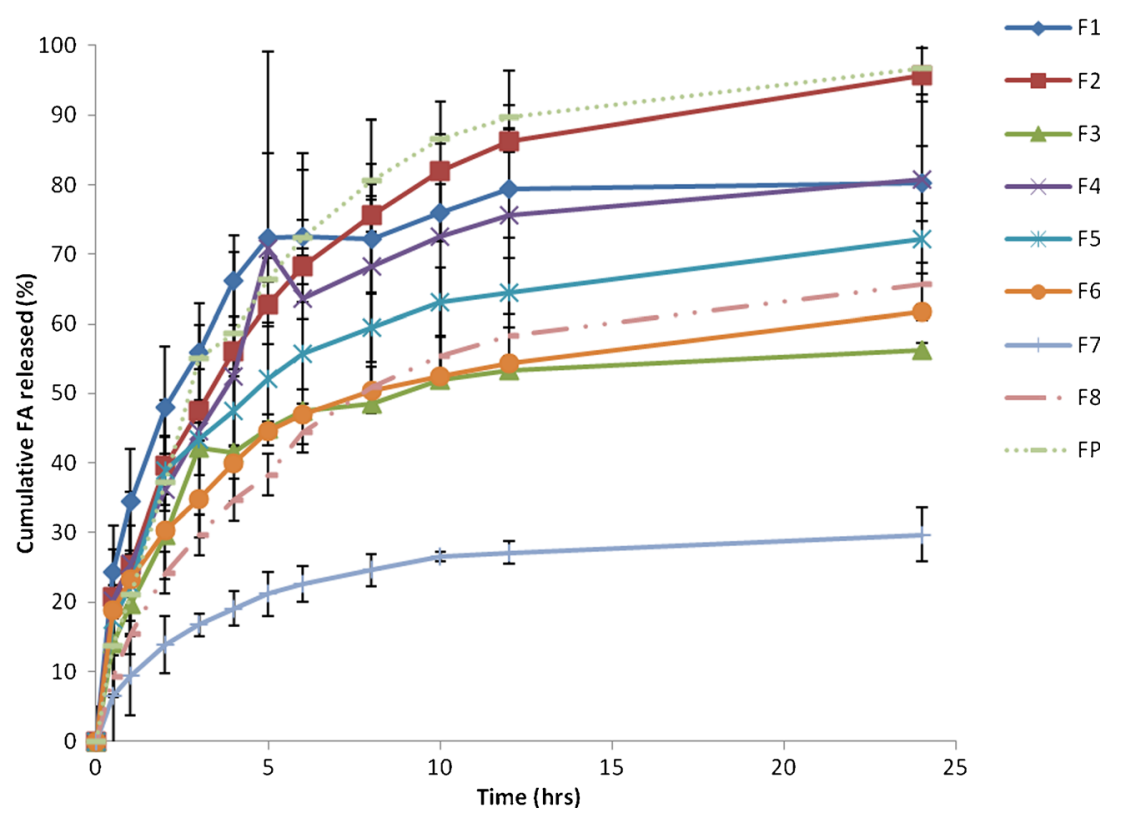

Fig. 2. Release profiles for fluocinolone acetonide $(F A)$ loaded PLGA nanoparticles as well as poloxamer system $(F P)$ 
Table IV. Release Data for Fluocinolone Acetonide Loaded PLGA Nanoparticles

\begin{tabular}{lllll}
\hline Formula code & $Q_{1 \mathrm{hr}}(\%)$ & $Q_{24 \mathrm{hr}}(\%)$ & $\mathrm{RE}(\%)$ & MRT (h) \\
\hline F1 & $12.61 \pm 0.87$ & $80.22 \pm 5.44$ & $71.78 \pm 6.83$ & $10.72 \pm 0.55$ \\
F2 & $25.30 \pm 5.75$ & $95.73 \pm 3.84$ & $76.30 \pm 2.53$ & $13.60 \pm 0.85$ \\
F3 & $19.75 \pm 8.94$ & $56.26 \pm 14.39$ & $48.45 \pm 13.22$ & $12.76 \pm 0.50$ \\
F4 & $18.12 \pm 10.39$ & $66.38 \pm 20.67$ & $53.93 \pm 19.41$ & $13.10 \pm 0.07$ \\
F5 & $22.34 \pm 3.43$ & $80.22 \pm 5.44$ & $59.21 \pm 8.19$ & $14.64 \pm 0.44$ \\
F6 & $15.40 \pm 2.06$ & $72.28 \pm 6.22$ & $41.81 \pm 11.81$ & $14.47 \pm 1.00$ \\
F7 & $9.39 \pm 5.69$ & $52.77 \pm 8.05$ & $24.39 \pm 7.32$ & $13.71 \pm 0.99$ \\
F8 & $15.40 \pm 5.42$ & $29.70 \pm 8.05$ & $27.27 \pm 15.47$ & $14.01 \pm 0.82$ \\
FP & $21.13 \pm 4.74$ & $96.79 \pm 2.07$ & $78.82 \pm 2.17$ & $13.34 \pm 0.30$ \\
\hline
\end{tabular}

$Q_{1 h r} \%$ cumulative amount of drug released after $1 \mathrm{~h}, Q_{24 h r} \%$ cumulative amount of drug released after $24 \mathrm{~h}, R E$ release efficiency, $M R T$ mean release time

mucoadhesion ability, stearlyamine (SA), a cationic surfactant, was added in different amounts $(5,10,25$ and $50 \mathrm{mg})$ to the organic phase during the preparation of F2. Prepared PLGA nanoparticles containing stearylamine were investigated; results are displayed in Table V.

It was found that by increasing the amount of stearylamine in $\mathrm{F} 2$ from 5 to $50 \mathrm{mg}$, an increase in the formulation viscosity occurred. Therefore, formulations prepared with $25 \mathrm{mg}$ stearylamine were highly viscous and formulations prepared with $50 \mathrm{mg}$ stearylamine formed a gel structure that was not easily removed from the flask during preparation. Therefore, both of them were excluded from further studies. It is well known that increasing the solution viscosity to a certain extent will increase eye retention but beyond this the opposite will occur due to the augmentation of reflex tearing and blinking. The high viscosity of applied preparations also has strong side effects like clogging of the puncti and canaliculi (40).

Addition of stearylamine, the cationic surface-modifying agent, can neutralize the negative charge of the PLGA polymer (Table V). This can be noticed in case of F2 formulation where its particle negative charge changed to a positive one after the addition of stearylamine. The difference in zeta potential between F2 and either SA1-F2 or SA2-F2 was significant $(p<0.05)$.

By the addition of 5 or $10 \mathrm{mg}$ of stearylamine to F2 (SA1-F2 and SA2-F2, respectively), the PLGA nanoparticles size did not

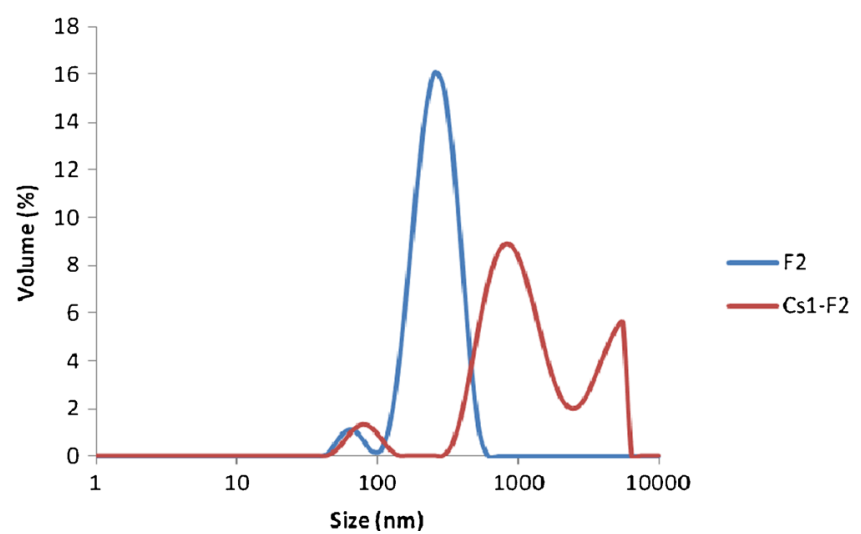

Fig. 3. Size distribution by volume for fluocinolone acetonide loaded PLGA nanoparticles (F2) and chitosan $\mathrm{HCl}$-coated fluocinolone acetonide loaded PLGA nanoparticles (Cs1-F2) show any change $(p>0.05)$ while PDI values increased significantly $(p<0.05)$, this may be due to the relocation of stearylamine on the surface of the particles increasing particleparticle interaction and forming some bridges between particles which can explain a broader size distribution compared to F2.

Stearylamine can act as both a complexing and emulsifying agent (41), which can increase drug retention in the smaller PLGA nanoparticles indicated in the supernatant; therefore, the EE increased in the supernatant and consequently decreased in PLGA nanoparticles present in the rest of the preparation (Table V).

Stearylamine-modified F2 were prepared aiming to explore the combined advantage of PLGA polymeric nanoparticulate system and impartment of surface charge for the improved delivery of fluocinolone acetonide to the eye. However, this goal seems not attained as the zeta potential was in small positive values for the stearylamine-modified F2 that were also accompanied with high PDI values; therefore, chitosan $\mathrm{HCl}$ surfacemodified F2 nanoparticles were prepared.

Chitosan and its derivatives are considerably explored in many researches aiming to modify and improve different delivery systems including colloidal systems. The high performance of chitosan-modified/coated particles has been proved in many of them (42-44), the cationic nature of chitosan as well as its distinguished mucoadhesive properties are the main reason. Such simple modifications for nanoparticles by coating them with chitosan can ensure a prolonged drug residence time in the site of action which can guarantee the improvement of the bioavailability of drugs (45).

Table VI shows the data for chitosan $\mathrm{HCl}$-coated $\mathrm{F} 2$; the formation of a chitosan $\mathrm{HCl}$ layer on the surface of the nanoparticles can be confirmed by comparing the particle size as well as the zeta potential values of nanoparticles before and after chitosan $\mathrm{HCl}$ coating. Size of nanoparticles in the whole preparation as well as small particles detected at the supernatant increased significantly $(p<0.05)$ by the addition of chitosan $\mathrm{HCl}$ and by increasing chitosan $\mathrm{HCl}$ concentration as well. The increase was probably due to a combination of adsorption and deposition of chitosan $\mathrm{HCl}$ molecules on the surface of the PLGA nanoparticles. Figure 3 demonstrate reprehensive curves for size distribution by volume for F2 and Cs1-F2 formulations.

The high increase in zeta potential values of all chitosan $\mathrm{HCl}$-coated F2 formulations compared to F2 is also clearly seen in the whole preparation and supernatant $(p<0.05)$; this is attributed to the cationic nature of chitosan $\mathrm{HCl}$ deposited on the surface of the PLGA nanoparticles.

The PDI values of F2 significantly increased by addition of different concentrations of chitosan $\mathrm{HCl}$ to it indicating a broader size distribution; this can be explained by coagulation, bridging and interconnection occurring between particles (Fig. 3).

By adding chitosan $\mathrm{HCl}$ to $\mathrm{F} 2$, the EE of the drug increased only in the small PLGA nanoparticles detected in supernatant $(p<0.05)$; this may be due to the deposition of the large chitosan $\mathrm{HCl}$ molecules on the surface of the particles inhibiting the leakage of the drug from it, in addition to possible fluocinolone acetonide-chitosan $\mathrm{HCl}$ interaction capturing the drug and preventing its possible diffusion out of the PLGA nanoparticles. However, this increase was concentration-independent so there was no increase in $\mathrm{EE}$ by increase of chitosan $\mathrm{HCl}$ concentration; this may be due to the saturation of the nanoparticles surfaces with chitosan $\mathrm{HCl}$ at a concentration of $0.1 \% w / v$, therefore 
Table V. Characterization of Stearyl Amine Containing F2 (SA-F2)

\begin{tabular}{|c|c|c|c|c|}
\hline Formula code & & $\mathrm{F} 2$ & $\mathrm{SA} 1-\mathrm{F} 2^{a}$ & $\mathrm{SA} 2-\mathrm{F} 2^{b}$ \\
\hline \multirow[t]{2}{*}{ Particle size (nm) } & Residue & $203.17 \pm 4.55$ & $192.03 \pm 7.38$ & $205.73 \pm 5.18$ \\
\hline & Supernatant & $178.6 \pm 2.26$ & $109.43 \pm 0.81$ & $113.93 \pm 0.50$ \\
\hline \multirow[t]{2}{*}{ PDI } & Residue & $0.38 \pm 0.02$ & $0.76 \pm 0.03$ & $0.77 \pm 0.05$ \\
\hline & Supernatant & $0.19 \pm 0.01$ & $0.23 \pm 0.01$ & $0.26 \pm 0.00$ \\
\hline \multirow[t]{2}{*}{ Zeta potential (mV) } & Residue & $-2.53 \pm 0.23$ & $2.64 \pm 0.15$ & $4.21 \pm 0.23$ \\
\hline & Supernatant & $4.87 \pm 0.58$ & $0.50 \pm 0.08$ & $1.62 \pm 0.61$ \\
\hline \multirow[t]{2}{*}{ Encapsulation efficiency (\%) } & Residue & $56.21 \pm 3.76$ & $34.65 \pm 2.32$ & $30.73 \pm 0.49$ \\
\hline & Supernatant & $6.48 \pm 3.77$ & $67.99 \pm 2.32$ & $72.39 \pm 0.49$ \\
\hline
\end{tabular}

$P D I$ polydispersity index

${ }^{a}$ PLGA nanoparticles prepared with $5 \mathrm{mg} \mathrm{SA}$

${ }^{b}$ PLGA nanoparticles prepared with $10 \mathrm{mg} \mathrm{SA}$

further increase of chitosan $\mathrm{HCl}$ concentration did not have any effect except for the increase in particle size reaching 2147.33 $\pm 125.63 \mathrm{~nm}$ for $0.50 \% \mathrm{w} / v$ chitosan $\mathrm{HCl}$ which is outside the framework of our goals.

As it showed from the previous investigations, sufficiently high zeta potential and EE values as well as reasonable particle size and PDI values were detected for Cs1-F2. Therefore, this formulation was selected for subsequent investigations.

Differential Scanning Calorimetry (DSC). DSC was carried out to characterize the physical state of drug in the selected PLGA nanoparticles system. The thermograms of the individual components, their physical mixtures, F2 and Cs1-F2 are shown in Fig. 4. The chitosan $\mathrm{HCl}$ thermogram shows an endothermic peak at 103.3 related to water loss associated with hydrogen bond to the chitosan $\mathrm{HCl}$ hydrophilic groups. Moreover, the chitosan $\mathrm{HCl}$ curve shows an endothermic peak at $222.2^{\circ} \mathrm{C}$, in addition to an exothermic one at $219.1^{\circ} \mathrm{C}$, referred to its degradation. Poloxamer gives rise to an endothermic peak at $64.1^{\circ} \mathrm{C}$ due to its melting point. The DSC curve for the PLGA present two peaks at around $46.49^{\circ} \mathrm{C}$ related to its glass transition. Fluocinolone acetonide exhibits a sharp endothermic peak at $272^{\circ} \mathrm{C}$ indicating its presence in a crystalline state. The DSC scans of the F2 and Cs1-F2 physical mixtures reveal the presence of an endothermic peak at 133.1 and $113.7^{\circ} \mathrm{C}$, respectively, which probably represent the fusion of the endothermic peaks of the associated polymers. Furthermore, the drug characteristic peak decreased in its intensity in the investigated physical mixtures which may be due to the dilution effect by the used polymers. The thermogram of chitosan $\mathrm{HCl}$-coated PLGA nanoparticles (Cs1-F2) was compared with that for the uncoated PLGA nanoparticles (F2) to detect the change in the PLGA nanoparticles state due to coating. F2 thermogram shows glass transition of the polymer at $40.48^{\circ} \mathrm{C}$ which was shifted to $47^{\circ} \mathrm{C}$ in case of Cs1-F2. This finding confirms the presence of entanglement between PLGA nanoparticles and its chitosan $\mathrm{HCl}$ coat indicating an efficient and stable coat (46). The endothermic characteristic peak of fluocinolone acetonide was not detected in the nanoparticles thermograms, suggesting the absence of the drug crystalline state and the formation of a molecular dispersion of the drug in the formulated colloids $(30,47)$.

Modified Surface PLGA Nanoparticles Shape. The transmission electron microphotographs of F2 and Cs1-F2 are shown in Fig. 5. F2 colloidal particles were uniform and almost spherical in shape, while Cs1-F2 seemed to be less uniform with clear depositions of the chitosan $\mathrm{HCl}$ on their surfaces as well as aggregation and inter-linking between particles with a less even particle size distribution compared to plain F2. This can explain the particle size and PDI results displayed before in Table VI and Fig. 3.

In Vitro Drug Release Studies for Chitosan HCl-Coated PLGA Nanoparticles. Release profiles of drug suspension, F2 and Cs1-F2 are displayed in Fig. 6. It can be clearly seen that Cs1-F2 attained the highest drug release profile. As expected,

Table VI. Characterization of Chitosan HCl-Coated F2 (Cs-F2)

\begin{tabular}{|c|c|c|c|c|c|}
\hline Formula code & & $\mathrm{F} 2$ & $\mathrm{Cs} 1-\mathrm{F} 2^{a}$ & $\mathrm{Cs} 2-\mathrm{F}^{b}$ & $\mathrm{Cs} 3-\mathrm{F} 2^{c}$ \\
\hline \multirow{4}{*}{$\begin{array}{l}\text { Particle size } \\
(\mathrm{nm}) \\
\text { PDI }\end{array}$} & Total preparation & $203.17 \pm 4.55$ & $779.53 \pm 34.09$ & $1107.00 \pm 68.09$ & $2147.33 \pm 125.63$ \\
\hline & Supernatant & $178.6 \pm 2.26$ & $442.20 \pm 3.82$ & $778.77 \pm 3.19$ & $1308.67 \pm 45.21$ \\
\hline & Total preparation & $0.38 \pm 0.02$ & $0.76 \pm 0.15$ & $0.61 \pm 0.07$ & $0.59 \pm 0.02$ \\
\hline & Supernatant & $0.19 \pm 0.01$ & $0.50 \pm 0.05$ & $0.55 \pm 0.02$ & $0.50 \pm 0.02$ \\
\hline \multirow{4}{*}{$\begin{array}{l}\text { Zeta potential } \\
(\mathrm{mV}) \\
\text { Encapsulation efficiency }(\%)\end{array}$} & Total preparation & $-2.53 \pm 0.23$ & $17.8 \pm 1.41$ & $33.53 \pm 3.08$ & $26.6 \pm 1.75$ \\
\hline & Supernatant & $4.87 \pm 0.58$ & $30.13 \pm 1.07$ & $34.17 \pm 0.91$ & $36.77 \pm 1.68$ \\
\hline & Residue & $56.21 \pm 3.76$ & $58.87 \pm 2.13$ & $58.55 \pm 3.19$ & $59.19 \pm 0.91$ \\
\hline & Supernatant & $6.48 \pm 3.77$ & $51.98 \pm 3.12$ & $47.79 \pm 3.15$ & $49.73 \pm 1.06$ \\
\hline
\end{tabular}

$P D I$ polydispersity index

${ }^{a}$ Prepared with $0.10 \% \mathrm{w} / v$ chitosan $\mathrm{HCl}$

${ }^{b}$ Prepared with $0.25 \% w / v$ chitosan $\mathrm{HCl}$

${ }^{c}$ Prepared with $0.50 \% \mathrm{w} / v$ chitosan $\mathrm{HCl}$ 


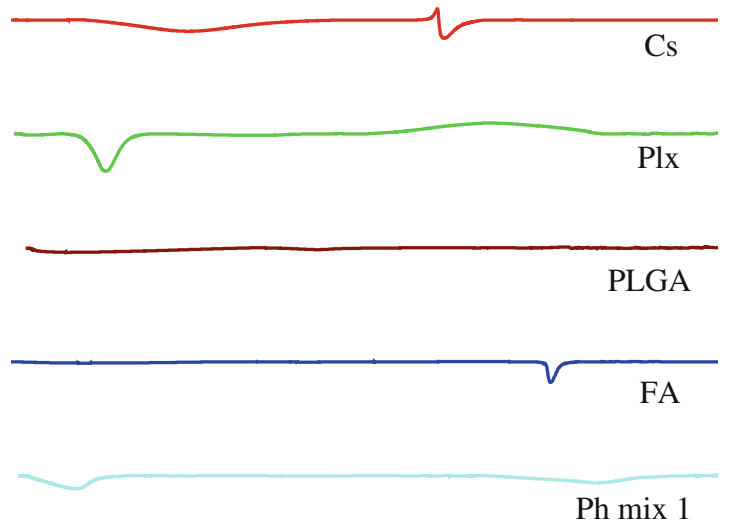

F2

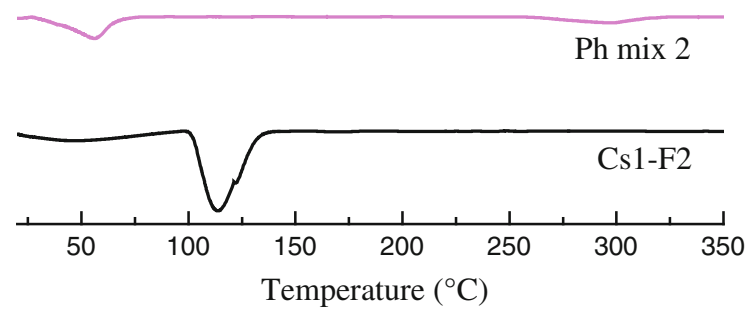

Fig. 4. DSC thermograms of chitosan $\mathrm{HCl}(C s)$, fluocinolone acetonide $(F A)$, poloxamer $407(P l x)$, poly(lactic-co-glycolic acid) type 5002A (PLGA), physical mixture of all components of F2 (Ph mix1), $F 2$ (drug loaded PLGA nanoparticles), physical mixture of all components of Cs1- F2 (Ph mix2) and Cs1-F2 (chitosan HCl-coated fluocinolone acetonide loaded PLGA nanoparticles)

drug suspension had the lowest $Q_{1 \mathrm{hr}}, Q_{24 \mathrm{hr}}$ and RE values $(p<0.05)$ when compared to that for F2 and Cs1-F2 as listed in Table VII. These results show the advantages of enlargement of surface contact area with external medium by decreasing formula particle size in case of F2 and Cs1-F2 (48). Both nanoparticulate systems showed a gradual increase in drug release, starting from the smaller PLGA nanoparticles as well as outer layers of the particles followed by larger particles and drug molecules embedded deeply.

XThe higher entrapment of drug in the supernatant of the selected chitosan $\mathrm{HCl}$ surface-modified PLGA nanoparticles (Cs1-F2; Table VI) is reflected in the obtained high release results. The increase of drug concentration in the nanoparticles can increase the concentration gradient which is an important release driving force and can, therefore, lead to increase the diffusion rate of the drug out of the preparation (47). Also, the diffusion of the drug out from the nanoparticles can be enhanced by the hydrophilic properties of chitosan $\mathrm{HCl}$ molecules attracting water to penetrate inside the nanoparticles.

Assessment of the Mucoadhesive Properties of SurfaceModified PLGA Nanoparticles

Chitosan is known to exhibit good mucoadhesive properties which prolong the residence time of nanoparticles at sites of drug action or absorption leading to an increment of both bioavailability and duration of action (49). The mucoadhesive properties of chitosan are mainly due to the molecular attractive forces resulted from the electrostatic interaction between the positively charged chitosan and the negatively charged mucosal surfaces. These properties are considered to be largely attributed to the strong charges, strong hydrogen bonding groups like $-\mathrm{OH}$, adequate chain flexibility and surface energy properties that help spreading on mucous (50). The mucoadhesive properties of the selected chitosan $\mathrm{HCl}$-coated PLGA nanoparticles were evaluated through measuring the effect of mucin (zeta potential value equals $-9.6 \mathrm{mV}$ ) on zeta potential and particle size values of the F2 and Cs1-F2. Zeta potential and particle size values of Cs1-F2 formulation changed from 17.8 to $1.9 \mathrm{mV}$ and from 779.5 to $1302.5 \mathrm{~nm}$, respectively, after incubation with mucin (Fig. 7). The obvious decrease in the zeta potential value and increase in particle size value was noticed in case of chitosan HCl-coated formulation (Cs1F2) but not in non-coated preparation (F2). This can be a great evidence of the good mucoadhesive properties of the prepared chitosan $\mathrm{HCl}$-coated PLGA nanoparticles.

In Vivo Evaluation of Selected PLGA Nanoparticles. The selected surface-modified PLGA nanoparticles (Cs1-F2) were compared with the unmodified PLGA nanoparticles (F2) regarding its ocular safety and tolerance as well as pharmacokinetics study. Rabbits were selected as an animal model for in vivo studies due to their convenience for formulations application as well as withdrawal of tears samples.

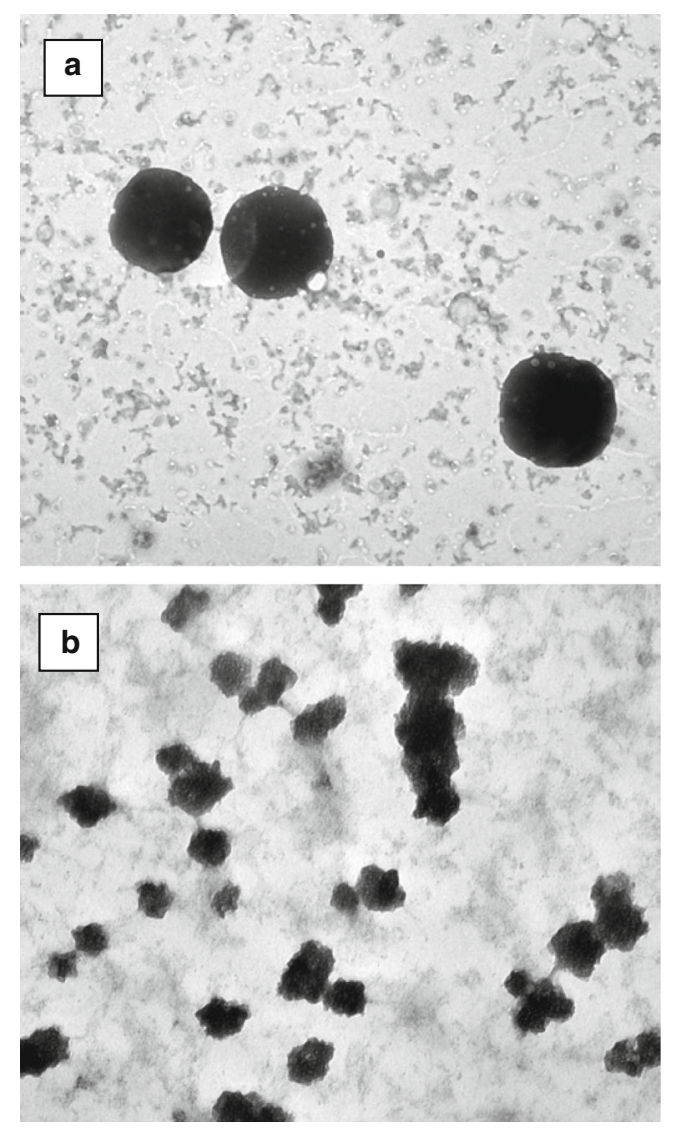

Fig. 5. TEM photographs of PLGA nanoparticles: a fluocinolone acetonide loaded PLGA nanoparticles (F2) and b chitosan $\mathrm{HCl}$ coated fluocinolone acetonide loaded PLGA nanoparticles (Cs1-F2) 


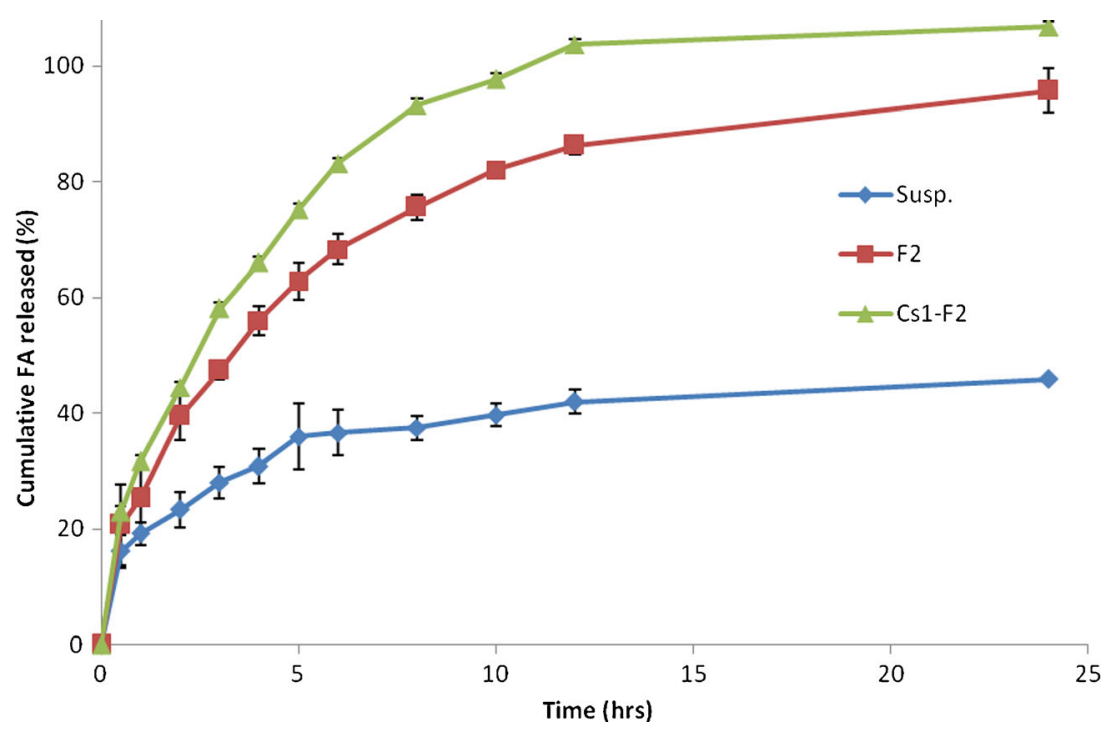

Fig. 6. Release profiles for fluocinolone acetonide (FA) suspension (Susp.), fluocinolone acetonide loaded PLGA nanoparticles (F2) and chitosan $\mathrm{HCl}$-coated fluocinolone acetonide loaded PLGA nanoparticles (Cs1-F2)

\section{Ocular Irritation Study}

Insufficient application of a certain medication by the patient is a great problem and is mainly due to the low clinical acceptance caused by some annoying side effects, such as irritation, burning, stinging and tearing. In general, the ophthalmic products must be well tolerated by the eyes and should not cause any inconvenience to the patients (30). For the unmodified and chitosan HCl-coated PLGA nanoparticles under study, ocular irritation test revealed that both were nonirritant and could be tolerated by the rabbit eye (average total score 0$)$.

\section{Pharmacokinetic Study}

To improve the delivery of the PLGA nanoparticles to the eye, the selected nanoparticles preparation was coated with the positively charged mucoadhesive polymer, chitosan $\mathrm{HCl}$. The successful surface modification of the nanoparticles with chitosan $\mathrm{HCl}$ was evident by the significant increase in the positive zeta potential values of the formulations as shown in Table VI. Binding of the applied nanoparticles formulation to the surface of the eye is thought to be of great relevance to prolonging its residence time resulting in higher bioavailability. To test such effect, the selected chitosan $\mathrm{HCl}$-coated

Table VII. Release Data for Drug Suspension, Formula F2 and Chitosan HCl-Coated F2 (Cs1-F2)

\begin{tabular}{lcccc}
\hline Formula & $Q_{1 \mathrm{hr}}(\%)$ & $Q_{24 \mathrm{hr}}(\%)$ & $\mathrm{RE}(\%)$ & MRT (h) \\
\hline $\begin{array}{l}\text { Drug } \\
\text { suspension }\end{array}$ & $19.19 \pm 2.84$ & $45.92 \pm 0.34$ & $38.30 \pm 1.82$ & $13.57 \pm 1.28$ \\
F2 & $25.30 \pm 5.75$ & $95.73 \pm 3.84$ & $76.30 \pm 2.53$ & $13.60 \pm 0.85$ \\
Cs1-F2 & $33.57 \pm 14.50$ & $106.71 \pm 1.17$ & $89.65 \pm 0.88$ & $12.83 \pm 0.24$ \\
\hline
\end{tabular}

$Q_{1 h r} \%$ cumulative amount of drug released after $1 \mathrm{~h}, Q_{24 h r} \%$ cumulative amount of drug released after $24 \mathrm{~h}, R E$ release efficiency, $M R T$ mean release time nanoparticles (Cs1-F2) was subjected to bioavailability study and compared to unmodified PLGA nanoparticles (F2).

The mean fluocinolone acetonide concentration in tears versus time curves following administration of F2 (group 1) and Cs1-F2 (group 2) are shown in Fig. 8.
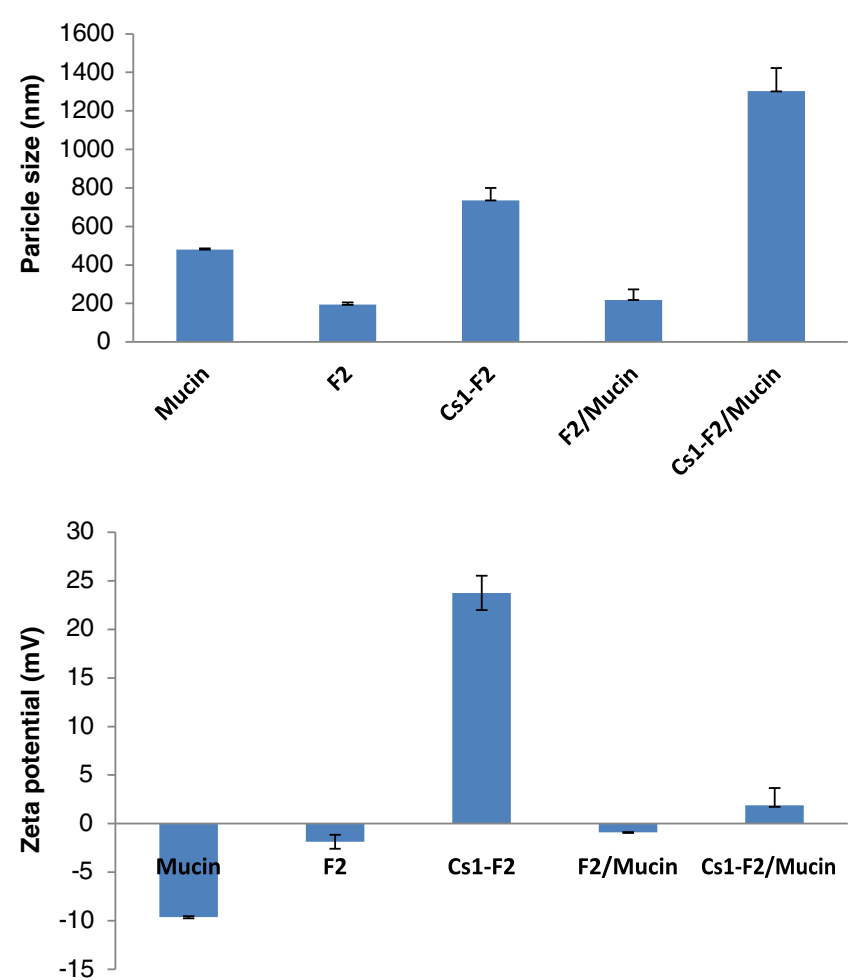

Fig. 7. The effect of adding mucin on the particle size and zeta potential value of fluocinolone acetonide loaded PLGA nanoparticles (F2) and chitosan HCl-coated fluocinolone acetonide loaded PLGA nanoparticles (Cs1-F2) 


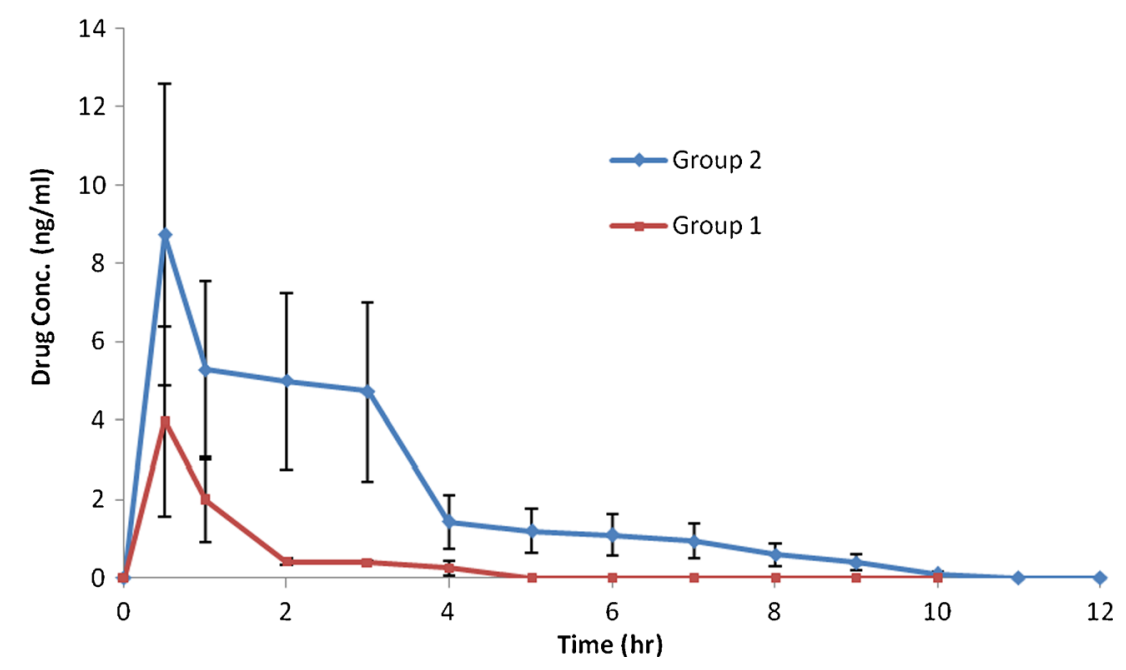

Fig. 8. Concentration of fluocinolone acetonide in tear samples of Albino rabbits as a function of time after administration of fluocinolone acetonide loaded PLGA nanoparticles (F2; group 1) and chitosan HCl-coated fluocinolone acetonide loaded PLGA nanoparticles (Cs1-F2; group 2)

The pharmacokinetic parameters derived from the tears concentration-time curves were calculated using WinNonlin ${ }^{\circledR}$ software (Pharsight Co., CA, USA) and are summarized in Table VIII. Both groups attained the highest drug level after $30 \mathrm{~min}$; this can reflect a beneficial rapid drug effect which is needed in many clinical cases. Group 2 showed a higher drug level $(p<0.05)$ all-over the study and remained higher up to $10 \mathrm{~h}$ compared to that for group 1 . On the other hand, in case of group 1, drug level was undetectable after $4 \mathrm{~h}$; hence, its AUC values were far lower than that for group $2(p<0.05)$. Furthermore, group 2 had larger MRT values compared to those for group 1 indicating longer residence time for chitosan $\mathrm{HCl}-$ coated PLGA nanoparticles in the eye. This can be explained by the mucoadhesive properties of chitosan $\mathrm{HCl}$ that is due to different physical and chemical chitosan-mucin interactions like hydrogen-bonding and ionic linking between the positive amino and the negative sialic acid groups of chitosan and mucin, respectively (12), which increased drug retention and bioavailability.

Table VIII. Pharmacokinetic Parameters of Fluocinolone Acetonide in Tears Samples of Albino Rabbits

\begin{tabular}{llr}
\hline Parameters & \multicolumn{2}{l}{ Tested formulation } \\
\cline { 2 - 3 } & $\mathrm{Gp} \mathrm{1(F2)}$ & $\mathrm{Gp} \mathrm{2} \mathrm{(Cs1-F2)}$ \\
\hline$C_{\text {max }}(\mathrm{ng} / \mathrm{ml})$ & $3.98 \pm 1.41$ & $8.72 \pm 3.86$ \\
$T_{\max }(\mathrm{h})$ & $0.50 \pm 0.00$ & $0.50 \pm 0.00$ \\
$t_{1 / 2}(\mathrm{~h})$ & $0.90 \pm 0.05$ & $1.73 \pm 0.09$ \\
$\mathrm{AUC}_{0-12 \mathrm{hr}}(\mathrm{ng} / \mathrm{ml} / \mathrm{h})$ & $4.39 \pm 1.02$ & $23.73 \pm 7.30$ \\
$\mathrm{AUC}_{0 \text {-inf }}(\mathrm{ng} / \mathrm{ml} / \mathrm{h})$ & $4.63 \pm 0.93$ & $24.21 \pm 6.54$ \\
$\mathrm{MRT}_{0-12 \mathrm{hr}}(\mathrm{h})$ & $1.13 \pm 0.07$ & $2.69 \pm 1.10$ \\
$\mathrm{MRT}_{0 \text {-inf }}(\mathrm{h})$ & $1.34 \pm 0.04$ & $2.88 \pm 1.27$ \\
\hline
\end{tabular}

Values are mean $(n=6) \pm \mathrm{SD}$
The inherent negative charge of the ocular structures can be exploited to increase drug ocular bioavailability by the incorporation of polymers of opposite charge within the preparation (12). Therefore, the surface charges of the nanoparticulate formulation under comparison can be an additional interpretation of the obtained results, as previously clarified; Table VI showed the reasonably high positive zeta potential values of Cs1-F2 compared to the negative value of F2.

Also, the penetration enhancing effect of chitosan can play a role by breaching corneal barriers. Dissociation of tight junctions of epithelial assemblies caused by chitosan without causing a permanent structural damage is a convincing mechanism of action $(12,26,51)$.

The higher amount of fluocinolone acetonide in Cs1-F2 when compared to F2 (Table VI) can also explain the higher drug level detected in tears, this is due to the increase of concentration gradient which increase drug release from the formulation to the external medium.

The innovated surface-modified PLGA nanoparticles (Cs1-F2) seem to attain an ideal pharmacokinetic profile ensuring rapid and prolonged in vivo drug delivery which can guarantee a rapid as well as extended clinical effect specially needed in certain cases.

\section{CONCLUSION}

An innovated simple method of nanoparticles preparation based on thin film hydration method was developed to prepare successful well-tolerated surface-modified PLGA nanoparticles. Also, this study demonstrates the importance of the use of the whole nanoparticles preparation without separation to avoid the exposure to the risk of loss of a certain amount of the drug which can be important in some cases. 


\section{ACKNOWLEDGMENTS}

The authors would like to thank the National Research Centre (NRC) and the Future University in Egypt (FUE), Cairo, Egypt, for their financial support.

\section{COMPLIANCE WITH ETHICAL STANDARDS}

Conflict of Interest The authors report no declarations of interest.

\section{REFERENCES}

1. Bucolo C, Drago F, Salomone S. Ocular drug delivery: a clue from nanotechnology. Front Pharmacol. 2012;3:188.

2. Sahoo SK, Dilnawaz F, Krishnakumar S. Nanotechnology in ocular drug delivery. Drug Discov Today. 2008;13(3-4):144-51.

3. Multicenter Uveitis Steroid Treatment (MUST) Trial Research Group. Benefits of systemic anti-inflammatory therapy versus fluocinolone acetonide intraocular implant for intermediate uveitis, posterior uveitis, and panuveitis: fifty-four-month results of the multicenter uveitis steroid treatment (MUST) trial and follow-up study. Ophthalmology. 2015;122(10):1967-75.

4. Katz M, Poulsen BJ. Corticoid, vehicle and skin interaction in percutaneous absorption. J Soc Cosmet Chem. 1972;23:565-90.

5. Dean E, Kumar RP. Surgical management of intraocular inflammation and infection. New Delhi: JP Medical Publishers; 2013.

6. Safety Data Sheet-British Pharmacopoeia. Safety data sheet according to 1907/2006/EC. 2014; Article 31: Page 5/7.

7. Schmit-Eilenberger VK. A novel intravitreal fluocinolone acetonide implant (Iluvien $((\mathrm{R}))$ ) in the treatment of patients with chronic diabetic macular edema that is insufficiently responsive to other medical treatment options: a case series. Clin Ophthalmol. 2015;9:801-11.

8. Cunha-Vaz J, Ashton P, Iezzi R, Campochiaro P, Dugel PU, Holz FG, et al. Sustained delivery fluocinolone acetonide vitreous implants: long-term benefit in patients with chronic diabetic macular edema. Ophthalmology. 2014;121(10):1892-903.

9. Parekh A, Srivastava S, Bena J, Albini T, Nguyen QD, Goldstein DA. Risk factors associated with intraocular pressure increase in patients with uveitis treated with the fluocinolone acetonide implant. JAMA Ophthalmol. 2015;133(5):568-73.

10. Dev S, McCallum RM, Jaffe GJ. Methotrexate treatment for sarcoid-associated panuveitis. Ophthalmology. 1999;106(1):111-8.

11. Hainsworth DP, Pearson PA, Conklin JD, Ashton P. Sustained release intravitreal dexamethasone. J Ocul Pharmacol Ther. 1996;12(1):57-63.

12. Nagarwal RC, Kant S, Singh PN, Maiti P, Pandit JK. Polymeric nanoparticulate system: a potential approach for ocular drug delivery. J Control Release. 2009;136(1):2-13.

13. Vafaei SY, Dinarvand R, Esmaeili M, Mahjub R, Toliyat T. Controlled-release drug delivery system based on fluocinolone acetonide-cyclodextrin inclusion complex incorporated in multivesicular liposomes. Pharm Dev Technol. 2014;26:1-7.

14. Danhier F, Ansorena E, Silva JM, Coco R, Le Breton A, Preat V. PLGA-based nanoparticles: an overview of biomedical applications. J Control Release. 2012;161(2):505-22.

15. Corrigan OI, Li X. Quantifying drug release from PLGA nanoparticulates. Eur J Pharm Sci. 2009;37(3-4):477-85.

16. $\mathrm{Mu} \mathrm{L}$, Feng SS. A novel controlled release formulation for the anticancer drug paclitaxel (Taxol): PLGA nanoparticles containing vitamin E TPGS. J Control Release. 2003;86(1):33-48.

17. Lemoine D, Preat V. Polymeric nanoparticles as delivery system for influenza virus glycoproteins. J Control Release. 1998;54(1):15-27.

18. Lin HR, Sung KC. Carbopol/pluronic phase change solutions for ophthalmic drug delivery. J Control Release. 2000;69(3):379-88.
19. Csaba N, Sanchez A, Alonso MJ. PLGA:poloxamer and PLGA:poloxamine blend nanostructures as carriers for nasal gene delivery. J Control Release. 2006;113(2):164-72.

20. Pinto Reis C, Neufeld RJ, Ribeiro AJ, Veiga F. Nanoencapsulation I. Methods for preparation of drug-loaded polymeric nanoparticles. Nanomedicine. 2006;2(1):8-21.

21. Gomez-Gaete C, Tsapis N, Besnard M, Bochot A, Fattal E. Encapsulation of dexamethasone into biodegradable polymeric nanoparticles. Int J Pharm. 2007;331(2):153-9.

22. Khan KA, Rhodes CT. Effect of compaction pressure on the dissolution efficiency of some direct compression systems. Pharm Acta Helv. 1972;47(10):594-607.

23. Gohel MC, Panchal MK. Novel use of similarity factors $f 2$ and $S$ $\mathrm{d}$ for the development of diltiazem $\mathrm{HCl}$ modified-release tablets using a 32 factorial design. Drug Dev Ind Pharm. 2002;28(1):7787.

24. Varshosaz J, Faghihian H, Rastgoo K. Preparation and characterization of metoprolol controlled-release solid dispersions. Drug Deliv. 2006;13(4):295-302.

25. Ammar HO, El-Nahhas SA, Ghorab MM, Salama AH. Chitosan/ cyclodextrin nanoparticles as drug delivery system. J Incl Phenom Macrocycl Chem. 2012;72(1):127-36.

26. de Campos AM, Diebold Y, Carvalho EL, Sánchez A, Alonso MJ. Chitosan nanoparticles as new ocular drug delivery systems: in vitro stability, in vivo fate, and cellular toxicity. Pharm Res. 2004;21(5):803-10.

27. Ammar HO, Salama H, Ghorab M, Mahmoud A. Nanoemulsion as a potential ophthalmic delivery system for dorzolamide hydrochloride. AAPS PharmSciTech. 2009;10(3):808-19.

28. Draize J, Woodward G, Calvery H. Methods for the study of irritation and toxicity of substances applied topically to the skin and mucous membranes. J Pharmacol Exp Ther. 1944;82:377-9.

29. Liu Z, Nie S, Guo H, Pan W, Li J. Effects of Transcutol P on the corneal permeability of drugs and evaluation of its ocular irritation of rabbit eyes. J Pharm Pharmacol. 2006;58(1):45-50.

30. Mahmoud AA, El-Feky GS, Kamel R, Awad GE. Chitosan/ sulfobutylether-beta-cyclodextrin nanoparticles as a potential approach for ocular drug delivery. Int J Pharm. 2011;413(1-2):22936.

31. Prabha S, Zhou WZ, Panyam J, Labhasetwar V. Sizedependency of nanoparticle-mediated gene transfection: studies with fractionated nanoparticles. Int J Pharm. 2002;244(12):105-15.

32. Salama HA, Mahmoud AA, Kamel AO, Abdel Hady M, Awad GA. Phospholipid based colloidal poloxamer-nanocubic vesicles for brain targeting via the nasal route. Colloids Surf B: Biointerfaces. 2012;100:146-54.

33. Larsson K. Cubic lipid-water phases: structures and biomembrane aspects. J Phys Chem. 1989;93(21):7304-14.

34. Gustafsson J, Ljusberg-Wahren H, Almgren M, Larsson K. Cubic lipid-water phase dispersed into submicron particles. Langmuir. 1996;12(20):4611-3.

35. Gustafsson J, Ljusberg-Wahren H, Almgren M, Larsson K. Submicron particles of reversed lipid phases in water stabilized by a nonionic amphiphilic polymer. Langmuir. 1997;13(26):6964-71.

36. Pawar D, Mangal S, Goswami R, Jaganathan KS. Development and characterization of surface modified PLGA nanoparticles for nasal vaccine delivery: effect of mucoadhesive coating on antigen uptake and immune adjuvant activity. Eur J Pharm Biopharm. 2013;85(3):550-9.

37. Janoria KG, Mitra AK. Effect of lactide/glycolide ratio on the in vitro release of ganciclovir and its lipophilic prodrug (GCVmonobutyrate) from PLGA microspheres. Int J Pharm. 2007;338(1-2):133-41.

38. Park TG. Degradation of poly(lactic-co-glycolic acid) microspheres: effect of copolymer composition. Biomaterials. 1995;16(15):1123-30.

39. Kamel R, Basha M, Abd El-Alim SH. Development of a novel vesicular system using a binary mixture of sorbitan monostearate and polyethylene glycol fatty acid esters for rectal delivery of rutin. J Liposome Res. 2013;23(1):28-36.

40. Barbault-Foucher S, Gref R, Russo P, Guechot J, Bochot A. Design of poly-epsilon-caprolactone nanospheres coated with 
bioadhesive hyaluronic acid for ocular delivery. J Control Release. 2002;83(3):365-75.

41. Rawat A, Majumder QH, Ahsan F. Inhalable large porous microspheres of low molecular weight heparin: in vitro and in vivo evaluation. J Control Release. 2008;128(3):224-32.

42. Yamamoto H, Kuno Y, Sugimoto S, Takeuchi H, Kawashima Y. Surface-modified PLGA nanosphere with chitosan improved pulmonary delivery of calcitonin by mucoadhesion and opening of the intercellular tight junctions. J Control Release. 2005;102(2):373-81

43. Kawashima Y, Yamamoto H, Takeuchi H, Kuno Y. Mucoadhesive DL-lactide/glycolide copolymer nanospheres coated with chitosan to improve oral delivery of elcatonin. Pharm Dev Technol. 2000;5(1):77-85.

44. Takeuchi H, Yamamoto H, Kawashima Y. Mucoadhesive nanoparticulate systems for peptide drug delivery. Adv Drug Deliv Rev. 2001;47(1):39-54.

45. Szymanska E, Winnicka K, Amelian A, Cwalina U. Vaginal chitosan tablets with clotrimazole-design and evaluation of mucoadhesive properties using porcine vaginal mucosa, mucin and gelatine. Chem Pharm Bull (Tokyo). 2014;62(2):160-7.

46. Kumar N, Khar P, Jain G, Kumar P, Malik A. Nanosuspension approach of acyclovir using het-cam method. IJDDMR. 2013;3:145-59.

47. Kamel R, Basha M, El Awdan S. Development and evaluation of long-acting epidural "smart" thermoreversible injection loaded with spray-dried polymeric nanospheres using experimental design. J Drug Target. 2013;21(3):277-90.

48. Gaumet M, Vargas A, Gurny R, Delie F. Nanoparticles for drug delivery: the need for precision in reporting particle size parameters. Eur J Pharm Biopharm. 2008;69(1):1-9.

49. He P, Davis SS, Illum L. In vitro evaluation of the mucoadhesive properties of chitosan microspheres. Int J Pharm. 1998;166:75-88.

50. Sinha VR, Singla AK, Wadhawan S, Kaushik R, Kumria R, Bansal K, et al. Chitosan microspheres as a potential carrier for drugs. Int J Pharm. 2004;274:1-33.

51. Nemoto E, Ueda H, Akimoto M, Natsume H, Morimoto $\mathrm{Y}$. Ability of poly-L-arginine to enhance drug absorption into aqueous humor and vitreous body after instillation in rabbits. Biol Pharm Bull. 2007;30(9):1768-72. 